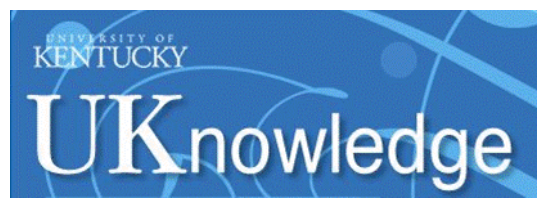

University of Kentucky

UKnowledge

5-16-2014

\title{
Subaru Spectroscopy and Spectral Modeling of Cygnus A
}

\author{
Matthew J. Merlo \\ Florida Institute of Technology \\ Erik S. Perlman \\ Florida Institute of Technology \\ Robert Nikutta \\ University of Kentucky, robert@pa.uky.edu \\ Christopher Packham \\ University of Texas at San Antonio \\ Moshe Elitzur \\ University of Kentucky, moshe@pa.uky.edu
}

See next page for additional authors

Follow this and additional works at: https://uknowledge.uky.edu/physastron_facpub

Part of the Astrophysics and Astronomy Commons, and the Physics Commons

Right click to open a feedback form in a new tab to let us know how this document benefits you.

\section{Repository Citation}

Merlo, Matthew J.; Perlman, Erik S.; Nikutta, Robert; Packham, Christopher; Elitzur, Moshe; Imanishi, Masatoshi; Levenson, N. A.; Radomski, James T.; and Aretxaga, Itziar, "Subaru Spectroscopy and Spectral Modeling of Cygnus A" (2014). Physics and Astronomy Faculty Publications. 186.

https://uknowledge.uky.edu/physastron_facpub/186

This Article is brought to you for free and open access by the Physics and Astronomy at UKnowledge. It has been accepted for inclusion in Physics and Astronomy Faculty Publications by an authorized administrator of UKnowledge. For more information, please contact UKnowledge@lsv.uky.edu. 


\section{Subaru Spectroscopy and Spectral Modeling of Cygnus A}

\section{Digital Object Identifier (DOI)}

https://doi.org/10.1088/0004-637X/788/1/6

\section{Notes/Citation Information}

Published in The Astrophysical Journal, v. 788, no. 1, 6, p. 1-9.

(C) 2014. The American Astronomical Society. All rights reserved.

The copyright holder has granted permission for posting the article here.

\section{Authors}

Matthew J. Merlo, Erik S. Perlman, Robert Nikutta, Christopher Packham, Moshe Elitzur, Masatoshi Imanishi, N. A. Levenson, James T. Radomski, and Itziar Aretxaga 


\title{
SUBARU SPECTROSCOPY AND SPECTRAL MODELING OF CYGNUS A
}

\author{
Matthew J. Merlo $^{1}$, Eric S. Perlman ${ }^{1}$, Robert Nikutta ${ }^{2,3}$, Christopher Packham ${ }^{4}$, Moshe Elitzur ${ }^{3}$,

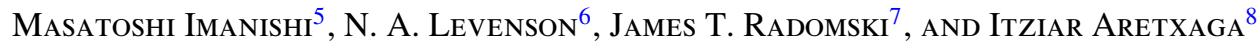 \\ ${ }^{1}$ Department of Physics and Space Sciences, Florida Institute of Technology, Melbourne, FL 32901, USA \\ 2 Department of Physics and Astronomy, University of Kentucky, Lexington, KY 40506-0055, USA \\ ${ }^{3}$ Departamento de Ciencias Físicas, Universidad Andrés Bello, Av. República 252, Santiago, Chile \\ ${ }^{4}$ Department of Physics and Astronomy, University of Texas at San Antonio, 1 UTSA Circle, San Antonio, TX 78249, USA \\ ${ }^{5}$ Subaru Telescope, 650 N. A'ohoku Place, Hilo, HI 96720, USA \\ ${ }^{6}$ Gemini Observatory, Casilla 603, La Serena, Chile \\ ${ }^{7}$ SOFIA/USRA, NASA Ames Research Center, Moffett Field, CA 94035, USA \\ ${ }^{8}$ Instituto Nacional de Astrofísica, Óptica y Electrónica, Calle Luis Erro 1, Sta. Ma. Tonantzintla, Puebla, Mexico \\ Received 2013 November 25; accepted 2014 April 1; published 2014 May 16
}

\begin{abstract}
We present high angular resolution $\left(\sim 00^{\prime} 5\right)$ MIR spectra of the powerful radio galaxy, Cygnus A (Cyg A), obtained with the Subaru telescope. The overall shape of the spectra agree with previous high angular resolution MIR observations, as well as previous Spitzer spectra. Our spectra, both on and off nucleus, show a deep silicate absorption feature. The absorption feature can be modeled with a blackbody obscured by cold dust or a clumpy torus. The deep silicate feature is best fit by a simple model of a screened blackbody, suggesting that foreground absorption plays a significant, if not dominant, role in shaping the spectrum of Cyg A. This foreground absorption prevents a clear view of the central engine and surrounding torus, making it difficult to quantify the extent the torus attributes to the obscuration of the central engine, but does not eliminate the need for a torus in Cyg A.
\end{abstract}

Key words: galaxies: active - galaxies: individual (Cygnus A) - infrared: galaxies

Online-only material: color figures

\section{INTRODUCTION}

Active galactic nuclei (AGNs) include many different object classes with diverse properties. They span as many as eight decades in luminosity, include objects with and without broad emission lines, and have a wide variety of spectral energy distributions (SED). The torus, proposed in the unified models of AGN (see, e.g., Antonucci 1993; Urry \& Padovani 1995 for reviews of the models), allows for different observed classes of objects to be explained by the viewing angle of the observer to the AGN, instead of physical differences between the classes. In order to determine whether the difference in class is due to the viewing angle to the torus or an actual physical difference in the object, comparison of the properties of the torus for each class is needed.

The size, structure, and geometry of the torus are not well constrained. Early modeling work proposed a uniform dust density for reasons of computational tractability (Krolik \& Begelman 1988; Pier \& Krolik 1992, 1993; Granato \& Danese 1994; Efstathiou \& Rowan-Robinson 1995). These models produced geometrically thick tori a few hundred parsecs in radius (Granato \& Danese 1994; Efstathiou et al. 1995). Since the energy that is absorbed from the central engine must be re-emitted at mid-infrared (MIR) wavelengths, the models were first tested against early Infrared Astronomical Satellite (IRAS) data and other far-infrared (FIR) data sets, with which they were consistent (Efstathiou et al. 1995; Granato et al. 1997). However, high angular resolution $(\leqslant 0$ '! 5$)$ MIR observations of AGN on $8 \mathrm{~m}$ class ground-based telescopes require much more compact tori, such as those found for NGC 4151 (upper limit on MIR size of $\lesssim 35$ pc; Radomski et al. 2003), Circinus (upper limit on MIR size of 12 pc; Packham et al. 2005), NGC 1068 (upper limit of $15 \mathrm{pc}$; Mason et al. 2006), Centaurus A (upper limit on MIR size of 3.5 pc; Radomski et al. 2008), and M87 (Perlman et al. 2001, 2007). More recently, MIR interferometry has found dust components at these scales for NGC 1068 (Jaffe et al. 2004), Centaurus A (Meisenheimer et al. 2007), Circinus (Tristram et al. 2007), and NGC 4151 (Burtscher et al. 2009). Larger samples of AGN observed with MIR interferometry also confirm compact tori for most objects in the samples (Tristram et al. 2009; Burtscher et al. 2013). Clumpy torus models fit the observed spectra and produce a torus scale consistent with the ground-based observations (Mason et al. 2006, 2009; Ramos Almeida et al. 2009; Nikutta et al. 2009; Ramos Almeida et al. 2011; Polletta et al. 2008; Hönig et al. 2008, 2010).

High resolution MIR imaging and spectroscopy have revealed important details of AGN, as seen in the subarcsecond variation detected for NGC 1068 (Mason et al. 2006) and Circinus (Roche et al. 2006). According to the unified scheme, there should be little difference in the torus structure between radio quiet (RQ) and radio loud (RL) AGN, except for the presence of radio jets. To date, nearly all high angular resolution observations of AGN have been done on RQ objects, which comprise $80 \%-90 \%$ of AGN. The first high angular resolution MIR imaging survey that included a significant number of RL AGN was presented by van der Wolk et al. (2010). We have also recently completed high angular resolution imaging observations of six more RL AGN. Mason et al. (2012) presents new imaging of four more RL AGN, and several more are presented in Asmus et al. (2011). High resolution imaging and spectroscopy has, until recently, only been done for two RL AGN: M87 (Perlman et al. 2001 (imaging); Perlman et al. 2007 (spectroscopy)) and Centaurus A (Meisenheimer et al. 2007 (spectroscopy); Radomski et al. 2008 (imaging)). Three other objects (3C445, González-Martín et al. 2013; IC5063, González-Martín et al. 2013; Ramos Almeida et al. 2011; NGC 1052, Mason et al. 2013) have been incorporated in samples within the last year.

Here we present high angular resolution MIR spectroscopy for Cygnus A (Cyg A). The redshift of Cyg A, $z=0.056$ (Stockton et al. 1994), corresponding to a distance of $247 \mathrm{Mpc}$ 

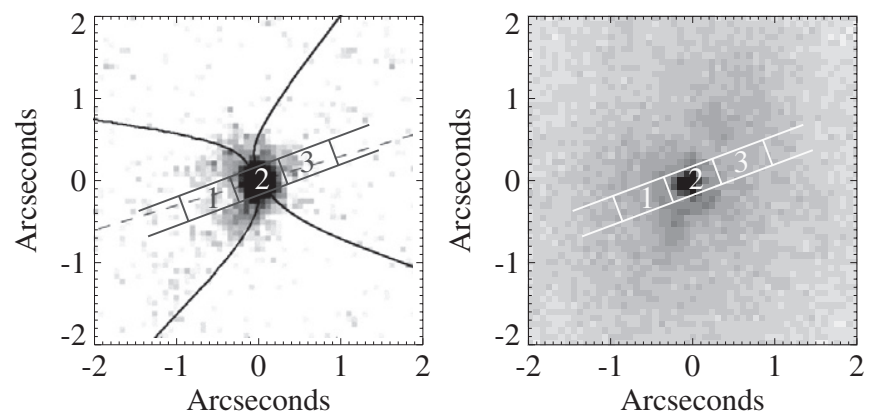

Figure 1. Left: $N$-band $\left(\lambda_{o}=10.8 \mu \mathrm{m}, \Delta \lambda=5.2 \mu \mathrm{m}\right)$ image of Cyg A with bicones (parabolas) and radio axis (dashed line) overplotted (Figure 4, Radomski et al. 2002). Right: $2.25 \mu \mathrm{m}$ NICMOS image of Cyg A from Tadhunter et al. (1999). These images show the unresolved nucleus and surrounding extended emission. Overplotted on the images are the slit and the extraction apertures used for our observations. Region 1 is the southeastern (SE) aperture, region 2 is the central aperture, and region 3 is the northwestern (NW) aperture. The total aperture is the combination of all three regions (see Section 2.1).

and a scale of $1^{\prime \prime}=1.1 \mathrm{kpc}$, makes it one of the closest powerful FR II radio galaxies. Because of its proximity and brightness, Cyg A is very well studied. Its radio jet structure and overall radio structure (Krichbaum et al. 1998; Carilli et al. 1999) have provided critical data for models of jets. X-ray spectroscopy found an intrinsic luminosity of the central core of $\sim 10^{44} \mathrm{erg} \mathrm{s}^{-1}$ and a large $\mathrm{H}$ I column density $\left(\sim 10^{23} \mathrm{~cm}^{-2}\right)$ (Young et al. 2002). Optical spectropolarimetry (Ogle et al. 1997) also found evidence of a powerful, obscured central engine. Furthermore, the polarization of narrow lines and the ratio of broad lines are consistent with scattering by dust. Chandra observations (Young et al. 2002) found evidence of a heavily obscured nucleus and a biconical soft $\mathrm{x}$-ray emission that is aligned with the bipolar cone seen in optical emission lines (Jackson et al. 1998) and nearinfrared (NIR; Tadhunter et al. 1999, 2000) observations. High resolution MIR imaging observations (Radomski et al. 2002; left panel of Figure 1) show an unresolved nucleus surrounded by extended emission that has a morphology consistent with the bipolar structure seen in optical and low resolution NIR emission lines. MIR spectroscopy presented in Imanishi \& Ueno (2000) found an absorption feature at $9.7 \mu \mathrm{m}$, consistent with absorption by dust near the central engine.

The paper is laid out as follows: in Section 2, we present MIR spectroscopic observations and data reduction of Cyg A made at the $8.2 \mathrm{~m}$ Subaru telescope, and archival data from other telescopes that will allow discussion of the broad band spectral properties of Cyg A. In Section 3, we discuss the spectral features of our spectra and compare with data from the Spitzer and Keck telescopes. In Section 4, we present and compare modeling of the MIR spectra, discuss the implications for previous work, and discuss the multi-wavelength SED. In Section 5, we discuss the overall significance of these observations and models.

\section{OBSERVATIONS AND DATA REDUCTION}

\subsection{Subaru Observations}

Spectroscopic observations of Cyg A were obtained UT 2005 April 27 on the Subaru telescope using the Cooled MidInfrared Camera and Spectrometer (COMICS; Kataza et al. 2000). COMICS is attached to the Cassegrain focus (Iye et al. 2004) and has five $320 \times 340 \mathrm{SiAs}$ impurity band conduction (IBC) detectors for spectroscopy. The plate scale is 0 '! 165 per pixel, which corresponds to a slit length of 39 '.6 on the sky. The detector uses correlated quadruple sampling for data readout (Sako et al. 2003). The observations were taken with the low-resolution $N$-band (NL) grating and 0 '.33 wide slit. This configuration disperses the entire $N$-band $(7.8-13.3 \mu \mathrm{m})$ onto one COMICS detector, with a spectral resolution of $\sim 250$ and a dispersion of $0.02 \mu \mathrm{m}$ per pixel. The observations utilized a chop throw of $10^{\prime \prime}$ at a frequency of $0.45 \mathrm{~Hz}$, at P.A. $=-69.5$ east of north, approximately aligned with the radio axis of Cyg A (Carilli et al. 1991). Chopping minimizes the background signal from the sky background, telescope thermal noise, and $1 / f$ detector noise. As is usual for spectroscopic observations with COMICS, the telescope was not nodded.

To reduce and calibrate the data, we followed the procedure outlined in Section 5.2 of the COMICS Data Reduction Manual version $2.1 .1,{ }^{9}$ using IRAF and the q_series software specifically designed for COMICS data.

Prior to extraction, for each raw data file, the average count rate in the slit was calculated and compared to the overall average for the night. Frames were rejected if the average count was less than the nightly average by at least $1 \sigma$ in both the raw and reduced data, likely due to adverse weather conditions. The total on-source exposure time after the removal of rejected data was $1618 \mathrm{~s}$.

First, the read-out noise pattern, created by taking the median of the readout channels from one of the unused detectors, was subtracted from the chopping-subtracted image. A dark frame taken during the observation was subtracted from a flat frame taken during the observation to form a dark-subtracted flat frame. The resulting image from the read-out noise subtraction was divided by the dark-subtracted flat frame. The resulting image was transformed to orthogonalize the dispersion and spatial axes and was then wavelength-calibrated. The resulting observed wavelengths were converted to the rest wavelengths for Cyg A. The spectra were then extracted from this frame. Observations of Vega, obtained directly before and after Cyg A, were used to remove telluric lines and to obtain an initial flux calibration.

Observing conditions were not photometric. The precipitable water vapor varied significantly between $3 \mathrm{~mm}$ and $7 \mathrm{~mm}$. Due to these conditions, a multi-step procedure was necessary to flux-calibrate these data. Initially, we fitted published MIR flux measurements for Vega (Cohen et al. 1992, 1999) with a power-law. The spectral data for Vega were then divided by this power-law and the Cyg A spectral data were divided by the result, in order to correct for atmospheric effects and instrument efficiency. We then multiplied this result by a correction factor (constant in $\lambda$ ) to match the total $N$-band flux of the spectrum to the $2^{\prime \prime}$ aperture flux for Cyg A in Radomski et al. (2002).

The spectra were extracted using several different apertures. Figure 1 shows the different apertures. A full width at half maximum (FWHM) of 0.'55 was measured for Vega. Three 0.66 (4 pixel) apertures, designated central, SE, and NW, were used to probe the nuclear and off-nuclear regions, where the radial profile $(\mathrm{FWHM} \sim 0$.'76) shows extended flux (Figure 2). A 1".98 (12 pixel) wide aperture, designated hereafter as the total aperture, includes the entire nuclear region (regions 1,2 , and 3 in Figure 1). This aperture combines our three smaller regions and extends to where the average counts fall to $1 \sigma$ above the background noise.

The extracted spectra are shown in Figure 3. In order to eliminate low signal-to-noise points at the short and long-

\footnotetext{
9 http://www.naoj.org/Observing/DataReduction/Cookbooks/ COMICS_COOKBOOK.pdf
} 


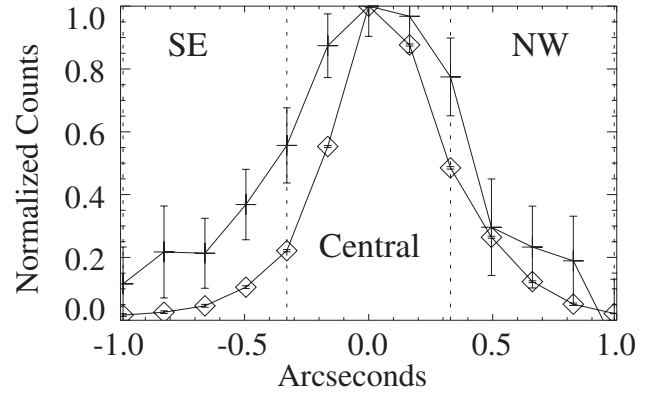

Figure 2. Radial profile plot for the spectral data for Cyg A (cross) and Vega (open diamond). We clearly detect flux from both the nucleus and extended structure.

wavelength ends of the spectra, points that were less than $3 \sigma$ above background for the total, central, and NW spectra, and less than $2 \sigma$ above the background for the SE spectrum are not plotted in Figure 3. The atmospheric ozone band between 9 and $10 \mu \mathrm{m}$ can still be seen, due to the poorer-than-average conditions. All Subaru spectra in Figure 3 have been binned in the spectral direction by 9 pixels to improve the signal-to-noise ratio $(\mathrm{S} / \mathrm{N})$ and to approximately match the spectral resolution $(\sim 50)$ of an earlier spectrum by Imanishi \& Ueno (2000).

Previous spectroscopic data, presented in Imanishi \& Ueno (2000), were taken on UT 1999 August 21 on the Keck I telescope using the Long Wavelength Spectrometer. They utilized a 0.5 wide slit and an N-wide filter $(8.1-13 \mu \mathrm{m})$, a total integration time of $1650 \mathrm{~s}$, and a spectral resolution of $\sim 50$. The Keck observations utilized an optimal extraction algorithm to extract the spectrum. Since the slit P.A. of the Imanishi \& Ueno (2000) spectrum is roughly perpendicular to our slit, P.A. $=31^{\circ}$ east of north, their spectrum includes regions of extended emission not covered by the COMICS data and does not include regions of extended emission covered by COMICS data. Due to possible uncertainties in flux calibration based on a slit spectrum, the authors matched the flux with previous $N$ band photometric data to much lower angular resolution (Rieke \& Low 1972) by multiplying by two. When this flux is compared with the flux observed for the Subaru and Spitzer spectra, it is necessary to remove this multiplication to reconcile the Imanishi \& Ueno (2000) flux with the Subaru and Spitzer flux, suggesting that the original calibration of the Imanishi \& Ueno (2000) data, without the factor of two, was accurate.

\subsection{Spitzer Observations}

Cyg A was observed with Spitzer using the Infrared Spectrograph (IRS) instrument (UT 2005 September 2) and the Multiband Imaging Photometer for SIRTF (MIPS) (UT 2004 October 17 ), as part of observing program ID 82 (PI: G. Rieke). The reduced IRS spectrum (Shi et al. 2006), plotted in Figure 4, was obtained from the authors. The MIPS images were obtained through the Spitzer data archive and were reduced through the standard Spitzer MIPS data pipeline for each wavelength.

The MIPS pipeline takes the raw data, corrects for instrument artifacts, subtracts a dark frame, and converts the data into flux units. For $24 \mu \mathrm{m}$ data, a flat field is applied and saturated pixels are replaced. For $70 \mu \mathrm{m}$ and $160 \mu \mathrm{m}$ data, an illumination correction is also applied. ${ }^{10}$ The post basic calibrated data pipeline takes the individual calibrated data frames and combines them into a mosaic. In order to conform to the recipe used in Shi et al. (2005) for MIPS measurements of other RL AGN, we used circular apertures of $\sim 15^{\prime \prime}$ at $24 \mu \mathrm{m}, \sim 30^{\prime \prime}$ at $70 \mu \mathrm{m}$, and $\sim 48^{\prime \prime}$ at $160 \mu \mathrm{m}$ to extract fluxes. For the $24 \mu \mathrm{m}$ data, the presence of other objects in the field of view made it necessary to extract several different circular background regions. Background subtraction on the $70 \mu \mathrm{m}$ and $160 \mu \mathrm{m}$ data was done using surrounding annuli $\left(\sim 40^{\prime \prime}-80^{\prime \prime}\right.$ for $70 \mu \mathrm{m}$ and $\sim 64^{\prime \prime}-128^{\prime \prime}$ for $160 \mu \mathrm{m})$. Aperture corrections were then applied to the data to correct for the amount of the point spread function outside the extraction aperture. For the $24 \mu \mathrm{m}$ and $70 \mu \mathrm{m}$ data, we used the corrections found in Shi et al. (2005) (1.146 for $24 \mu \mathrm{m}, 1.3$ for

\footnotetext{
10 http://irsa.ipca.caltech.edu/data/SPITZER/docs/mips/ mipsinstrumenthandbook/45/, see specifically Table 4.3.
}
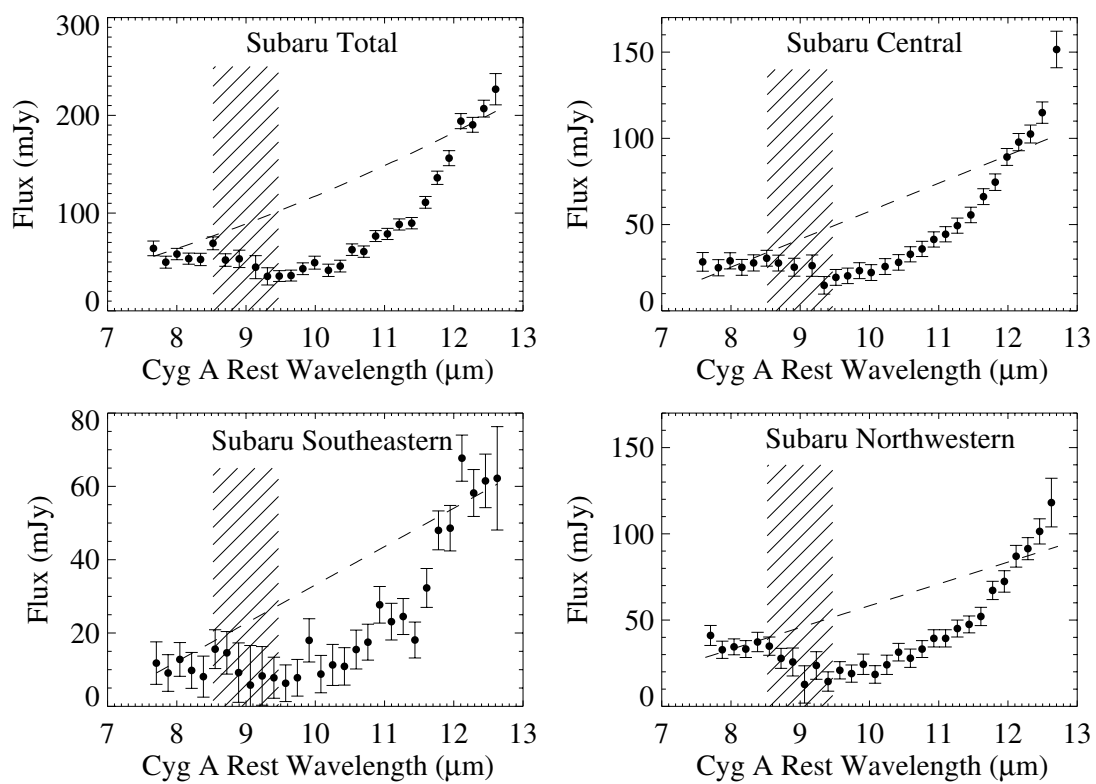

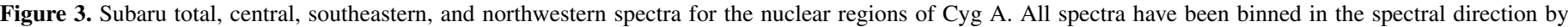

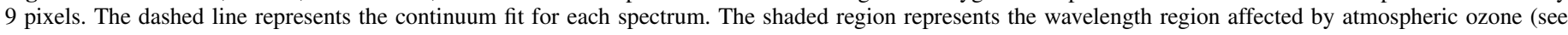
Sections 2.1 and 3 for discussion). 

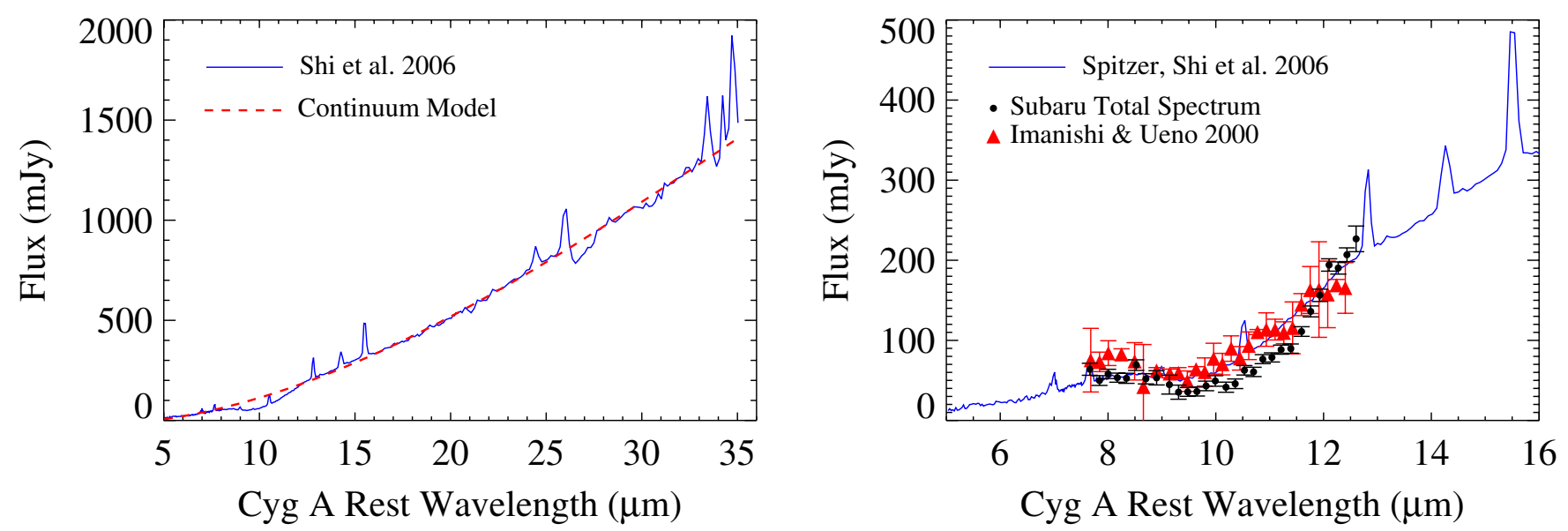

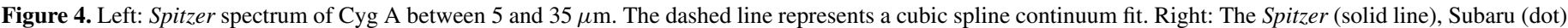

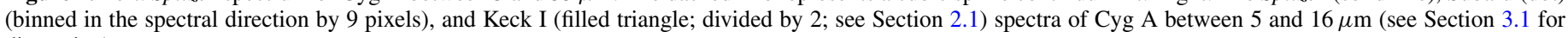
discussion).

(A color version of this figure is available in the online journal.)

Table 1

Multiwavelength Observations

\begin{tabular}{|c|c|c|c|c|c|c|c|}
\hline Telescope & Instrument & Filter & $\begin{array}{c}\text { Observation } \\
\text { Date (UT) }\end{array}$ & $\begin{array}{c}\text { Exposure } \\
\text { Time (s) }\end{array}$ & $\begin{array}{c}\text { Wavelength } \\
(\mu \mathrm{m})\end{array}$ & $\begin{array}{l}\text { Flux }^{\mathrm{a}} \\
(\mu \mathrm{Jy})\end{array}$ & Reference \\
\hline Chandra & ACIS & $\ldots$ & 2000 May 26 & 9228 & $0.000138-0.00177$ & $\ldots$ & Young et al. (2002) \\
\hline HST & ACS:SBC & F140LP & 2009 Apr 9 & 1206 & 0.153 & $<8$ & $\ldots$ \\
\hline$H S T$ & STIS: NUVMAMA & F25SRF2 & 2000 Jun 25 & 2160 & 0.230 & $<4$ & Allen et al. (2002) \\
\hline$H S T$ & FOC F/96 COSTAR & F342W & 1994 Apr 18 & 1195.875 & 0.340 & $20 \pm 1$ & $\ldots$ \\
\hline$H S T$ & FOC F/96 COSTAR & F372M & 1994 Apr 19 & 744.875 & 0.371 & $20 \pm 1$ & $\ldots$ \\
\hline$H S T$ & NICMOS:NIC2 & F222M & 1997 Dec 15 & 960 & 2.25 & $2000 \pm 100$ & Tadhunter et al. (1999) \\
\hline Subaru & IRCS & $\mathrm{L}$ grism & 2005 May 29 & 1440 & $2.8-4.1$ & $\ldots$ & Imanishi (2006) \\
\hline Spitzer & MIPS & $24 \mu \mathrm{m}$ & 2004 Oct 17 & 168 & 23.7 & $650 \pm 19$ & $\ldots$ \\
\hline Spitzer & MIPS & $70 \mu \mathrm{m}$ & 2004 Oct 17 & 168 & 71 & $1920 \pm 25$ & $\ldots$ \\
\hline Spitzer & MIPS & $160 \mu \mathrm{m}$ & 2004 Oct 17 & 178 & 156 & $480 \pm 22$ & $\ldots$ \\
\hline Keck II & OSCIR & $\mathrm{N}$ & 1998 May 9 & 240 & 10.8 & $104 \pm 3$ & Radomski et al. (2002) \\
\hline Keck II & OSCIR & IHW18 & 1998 May 9 & 180 & 18.2 & $319 \pm 27$ & Radomski et al. (2002) \\
\hline JCMT & SCUBA & Multiple & Multiple & Multiple & Multiple & $\ldots$ & Robson et al. (1998) \\
\hline
\end{tabular}

Note. ${ }^{\text {a }}$ See Section 2.3 for discussion.

$70 \mu \mathrm{m})$. For the $160 \mu \mathrm{m}$ data, we applied a correction factor of 1.6, as recommended in the MIPS Instrument Handbook. ${ }^{11}$

\subsection{Multiwavelength Observations}

An $L$-band (2.8-4.1 $\mu \mathrm{m})$ observation, presented in Imanishi (2006), was taken with the Infrared Camera and Spectrograph (IRCS) on Subaru on UT 2005 May 29. The observation utilized a 0.9 slit with a P.A. $=0$, had an integration time of 24 minutes, and used the standard nodding technique. The data was reduced using IRAF to subtract the nod position from each other, combine these subtracted images, and divide by a flat frame. For bad pixels or pixels effected by cosmic rays, the surrounding pixels were used to interpolate a value for the affected pixel. The data was then wavelength and flux calibrated and binned to achieve an $\mathrm{S} / \mathrm{N}$ of 10 or greater.

Chandra observations (OBSID 1701, PI: Andrew Wilson) were taken on UT 2000 May 26, and were previously presented by Young et al. (2002). The observations had an on-source time of $9228 \mathrm{~s}$ and a circular extraction aperture of 2.5 in diameter

11 http://irsa.ipca.caltech.edu/data/SPITZER/docs/mips/
(Young et al. 2002). To obtain flux measurements, we used the spectroscopic models of Young et al. (2002), which combined several different spectral components to model the Chandra spectrum. These components included a heavily absorbed power law, a power law plus narrow emission lines absorbed by a galactic column density, and a neutral iron fluorescence line. The fluxes predicted by these models were obtained using Xspec ver. 12.7.0.

All Hubble Space Telescope (HST) imaging observations of Cyg A were obtained from the $H S T$ data archive. In Table 1, we list the instruments, dates, and other relevant information. The standard pipeline reductions were used for all the data. Details of the data reduction pipeline can be found in the respective instrument handbooks. ${ }^{12}$ Fluxes were extracted from circular $2^{\prime \prime}$ regions to approximately match the aperture of the Subaru total spectrum. Background fluxes were also obtained from a circular $2^{\prime \prime}$ region that was far enough from each source so that

\footnotetext{
12 http://documents.stsci.edu/hst/wfpc2/documents/handbooks/dhb/wfpc2_ cover.html

http://www.stsci.edu/hst/acs/documents/handbooks/currentDHB/acs_cover.html http://www.stsci.edu/hst/nicmos/documents/handbooks/DataHandbookv8/ http://www.stsci.edu/documents/dhb/webvol2/archive_dhbTOC.doc.html http://www.stsci.edu/hst/stis/documents/handbooks/currentIHB/cover.html
} 

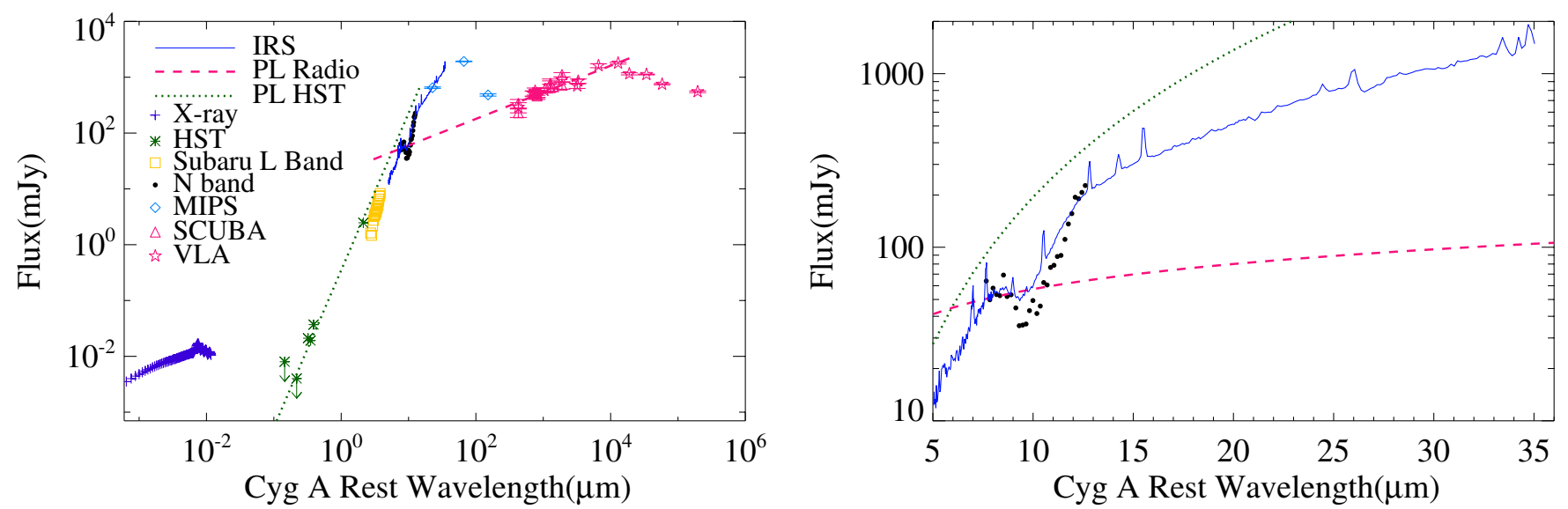

Figure 5. Left: spectral energy distribution of Cyg A including Chandra (cross), HST (asterisk), Subaru L band (open square), Subaru total (dot), Spitzer IRAS (solid line), Spitzer MIPS (open diamond), VLA (open star), and SCUBA (open triangle) data. The dotted and dashed lines represent a power law fit to the HST and radio data, respectively. Right: a larger view of the 5-36 $\mu \mathrm{m}$ region (see Section 2.3 for discussion).

(A color version of this figure is available in the online journal.)

no extended emission was included. All fluxes were aperturecorrected for each individual instrument, as recommended in their instrument handbooks ${ }^{12}$. Data for WFPC2/F555W are available, but were not included due to contamination from emission lines (mainly [O III]).

The 10.7 and $18 \mu \mathrm{m}$ data points were obtained from Radomski et al. (2002). These observations were taken on the Keck II $10 \mathrm{~m}$ telescope using the mid-infrared camera/spectrometer OSCIR on UT 1998 May 9. The $N\left(\lambda_{0}=10.8 \mu \mathrm{m}, \delta \lambda=5.2 \mu \mathrm{m}\right)$ and IHW18 $\left(\lambda_{0}=18.2 \mu \mathrm{m}, \delta \lambda=1.7 \mu \mathrm{m}\right)$ filters were used, with a total on-source time of $240 \mathrm{~s}$ for $N$-band and $180 \mathrm{~s}$ for IHW18. The reduced images were flux calibrated using observations of standard stars. The flux was measured using a $2^{\prime \prime}$ aperture.

The sub-millimeter flux data were taken on the James Clerk Maxwell Telescope (JCMT), using the Submillimetre CommonUser Bolometer Array (SCUBA) instrument. The data was obtained from Table 1 of Robson et al. (1998). Only the core observations from this table were used. The extraction region for these data was $6^{\prime \prime} \times 6^{\prime \prime}$, centered on the radio core position. The Very Large Array (VLA) flux data were presented in Carilli et al. (1999).

\subsection{The SED and Components of MIR Nuclear Emission}

In Figure 5, multiwavelength flux data (Section 2.3) for the nucleus of Cyg A is plotted. As discussed above, the dominant component of MIR emission arises from heated dust. This thermal component from dust is the major contributor to the MIR spectra and the MIPS points seen in Figure 5. The MIPS points suggest the presence of cooler dust at larger radii.

The SCUBA and VLA radio data are fit with a power law (dashed pink line in Figure 5) of the form $F(\mathrm{Jy})=$ $(0.019 \pm 0.003) \lambda^{0.48 \pm 0.02}$, with $\lambda$ in $\mu \mathrm{m}$. This represents the synchrotron emission from the base of the jet. Figure 5 indicates that synchrotron emission from the jet (which is represented by the power law at low frequency plotted there) dominates in the radio and likely does account for a non-negligible fraction of the emission beyond 50-100 $\mu \mathrm{m}$. The synchrotron emission severely underestimates MIR flux at $\lambda>10 \mu \mathrm{m}$ and overestimates flux at $\lambda<10 \mu \mathrm{m}$. Thus, a break in the synchrotron emission at $\lambda \sim$ tens of microns is required. This agrees with the analysis of Meisenheimer et al. (2001),
Table 2

Apparent $\tau_{9.7}$ for $10 \mu \mathrm{m}$ Silicate Feature

\begin{tabular}{lcc}
\hline \hline Telescope & Optical Depth & Uncertainty \\
\hline Spitzer & 0.6 & 0.04 \\
Subaru total & 0.9 & 0.1 \\
Subaru central & 0.9 & 0.1 \\
Subaru northwest & 1.0 & 0.2 \\
Subaru southeast & 1.0 & 0.2 \\
Keck I & 0.6 & 0.1 \\
\hline
\end{tabular}

specifically their Figure 6. A similar conclusion was also reached by Privon et al. (2012) using Spitzer data.

The HST data are fit with a power law, (dotted green line in Figure 5) of the form $F(\mathrm{Jy})=\left(3.0 \pm 0.4 \times 10^{-4}\right) \lambda^{2.8 \pm 0.7}$. This represents a combination of the big blue bump feature seen in AGN spectra, emission from hot dust, and stellar contribution. The $K$ - and $L$-band data may also represent a stellar contribution, however the $L$-band data does not have any polycyclic aromatic carbon (PAH) features, which are signs of starbursts. This lack of PAH features is also seen in the Spitzer and Subaru spectra. The absence of PAH features does not necessarily mean there is no starburst activity, as the AGN can destroy the PAH. The $K$ and $L$-band do represent a distinct component, however, as their slopes do not match the HST data or the MIR spectral data.

\section{ANALYSIS AND RESULTS}

In order to model the continuum emission in each of the MIR spectra, splines were fit to the data. For the Spitzer spectrum, a cubic spline was fit to estimate the continuum shape (dashed line in Figure 4). Since the Subaru total spectrum agrees well with the Spitzer spectrum at the red and blue ends (see Figure 4), the well-defined continuum fit for Spitzer is used. For the remaining Subaru spectra and the Imanishi \& Ueno (2000) spectrum, a linear spline was fit for each individual spectrum, as these spectra do not match the Spitzer spectrum (dashed lines in Figure 3).

\subsection{MIR Silicate Feature}

The dominant spectral feature between 7 and $13 \mu \mathrm{m}$ is silicate absorption ( $\sim 9 \mu \mathrm{m}$ to $\sim 12 \mu \mathrm{m}$; see Figures 3 and 4 ). Table 2 
shows the apparent $\tau_{9.7}$ in the silicate feature of each spectrum. This was calculated using

$$
\tau=-\ln \left(\frac{F_{\text {spec }}}{F_{\text {cont }}}\right),
$$

where $F_{\text {spec }}$ (flux of each spectrum) and $F_{\text {cont }}$ (flux of the corresponding spline fit for each spectrum) are averages between 9.5 and $10.3 \mu \mathrm{m}$. The optical depths found in the total and central Subaru spectra are the same within errors and show a larger $\tau_{9.7}$ than seen in the Spitzer spectrum (Figure 4, Table 2), a result of Subaru's higher angular resolution, which gives a less contaminated sight-line to the central regions of the galaxy. Our $\tau_{9.7}$ values agree with the findings of Imanishi \& Ueno (2000; i.e., $\tau_{9.7} \sim 1$ ), although they used a different technique (which does not require fitting the continuum) to find the optical depth due to their lower $S / N$. If their data is analyzed using a linear spline continuum fit and the above procedure, an optical depth comparable with Spitzer is found (Table 2). Because the slits for Imanishi \& Ueno (2000) and our spectra are almost perpendicular, comparing the spectra gives a view of two different regions of Cyg A. Differences in the spectral shape between the two spectra may be due to the different regions covered by the spectra. Our slit is along the radio axis of Cyg $\mathrm{A}$, which samples the core and the ionization cone region (see Figure 1), whereas the Imanishi \& Ueno (2000) slit is perpendicular to the radio axis, which samples the core but does not include the ionization cone region.

\section{MODELING OF THE MIR SPECTRA}

Modeling of the MIR spectra gives insight into the inner region of the AGN. Absorption and re-emission of radiation by dust surrounding the central engine produces MIR emission. Therefore, modeling of MIR spectra probes the configuration of that dust and can help determine if spectra are the result of a dusty torus, dust not associated with the AGN, or MIR emission other sources (i.e., star formation). In order to probe these possibilities, we present two different models fitted to the Subaru spectra.

\subsection{CLUMPY Model}

The Subaru spectra present an opportunity to apply a torus model to spectroscopic data for a high power RL AGN. In order to fully utilize the high angular resolution of our Subaru spectra, we chose to model the Subaru central spectrum and the $18 \mu \mathrm{m}$ point from Radomski et al. (2002) with the CLUMPY model (Nenkova et al. 2008a, 2008b). Emission lines and the ozone band in the spectrum were removed when fitting the model, as these features cannot be modeled by CLUMPY. The spectrum is also re-binned to fit the CLUMPY grid, which has a step size of $0.25 \mu \mathrm{m}$ between $6.0-8.5 \mu \mathrm{m}$ and $11.5-20.0 \mu \mathrm{m}$ and a step size of $0.1 \mu \mathrm{m}$ between 8.5 and $11.5 \mu \mathrm{m}$.

CLUMPY models the statistical properties of an ensemble of discrete clouds of dust surrounding the central engine of an AGN in a toroidal structure. The number of clouds observed along the line of sight determines the total obscuration (Nenkova et al. 2002, 2008a, 2008b; Hönig et al. 2006; Schartmann et al. 2008). CLUMPY has six free parameters, $i$ (viewing angle), $\tau_{V}$ (optical depth of each cloud), $q$ (radial density profile index, i.e., $r^{-q}$ is how the density of clouds behaves with respect to radius from the central engine), $N_{0}$ (number of clouds along an equatorial line of sight), $\sigma$ (the angular thickness of the torus), and $Y$ (the radial thickness of the torus, i.e., the ratio between the
Table 3

CLUMPY Model Initial Parameter Ranges

\begin{tabular}{lccr}
\hline \hline Parameter & Symbol & Interval & Median $^{\mathrm{a}}$ \\
\hline Viewing angle & $i$ & {$\left[50^{\circ}, 90^{\circ}\right]$} & $88_{-5}^{+2}$ \\
Optical depth per cloud & $\tau_{V}$ & {$[5,150]$} & $40_{-11}^{+9}$ \\
Radial density profile index & $q$ & {$[0.0,3.0]$} & $1.4_{-0.4}^{+0.1}$ \\
Number of clouds & $N_{0}$ & {$[1,15]$} & $14.9_{-0.4}^{+0.1}$ \\
along equatorial line of sight & & & \\
Torus angular thickness & $\sigma$ & {$\left[30^{\circ}, 40^{\circ}\right]$} & $39.8_{-0.5}^{+0.2}$ \\
Torus radial thickness & $Y$ & {$[5,100]$} & $94.5_{-10.3}^{+5.5}$ \\
\hline
\end{tabular}

Note. ${ }^{\text {a }}$ See Section 4.1 for discussion about the constraints on $\sigma, i$, and $N_{0}$ and for discussion of fitting.

inner radius, defined by the sublimation radius for the dust, and the outer radius), which describe the structure of the torus and of the individual clouds within it (Table 3; see Nenkova et al. 2008a, 2008b for a full description). The SEDs resulting from many combinations of model parameter values are stored in a public database for later use. ${ }^{13}$

To model our observed data with CLUMPY, we used Bayesian analysis with Markov chain Monte Carlo sampling of the posteriors. A full description of the techniques and tools is given in R. Nikutta et al. (2013, in preparation).

In Table 3, we list the model parameters and their allowed ranges. These represent standard CLUMPY input ranges, except for the viewing angle $i\left(50^{\circ}<i<90^{\circ}\right.$; Tadhunter et al. $2003)$ and angular thickness $\sigma\left(30^{\circ}<\sigma<40^{\circ}\right.$; Jackson et al. 1996; Ogle et al. 1997; Tadhunter et al. 1999), where previous observations provide independent constraints for these parameters. By constraining the parameters with previous observations, it is possible to eliminate some of the degeneracy inherent when fitting a six parameter model, as well as producing a model that does not give nonphysical parameters. The upper limit for $N_{0}$ is set at 15 as higher values produce a narrow IR bump peaking beyond $60 \mu \mathrm{m}$, which has not been observed in AGN SEDs (Nenkova et al. 2008b).

We find that drawing $10^{6}$ samples yields very smooth posteriors and converged Markov chains. The one-dimensional, marginalized posteriors are obtained from the full sixdimensional posterior by marginalizing over the other five parameters. Once such posteriors have been obtained, the $1 \sigma$ and $2 \sigma$ error ranges are simply the intervals around the median that contain $68.3 \%$ or $95.4 \%$, respectively, of the total of the cumulative distribution function of the posterior. We present the posteriors for fitting our Subaru data in Figure 6.

Since the CLUMPY models cover a much larger wavelength range than our Subaru spectra, we attempted to include $K$ and $L$ band data to constrain the short wavelength end of the model. These data caused the $\chi^{2}$ to become much worse, however, and these data points are likely part of a spectral component other than the torus.

The best fit CLUMPY model, fitted between 7.75 and $12.5 \mu \mathrm{m}$, yields a reduced $\chi^{2}=2.32$ and a $p$-value $=0.0002$, with 25 degrees of freedom. If the $18 \mu \mathrm{m}$ point is included in the fit, the best model yields a reduced $\chi^{2}=2.27$ and $p=0.0002$, with 26 degrees of freedom. The low $p$-values for both these fits suggest that the fits are not satisfactory. The results of our modeling are shown in Figures 6 and 7.

\footnotetext{
13 www.pa.uky.edu/clumpy
} 

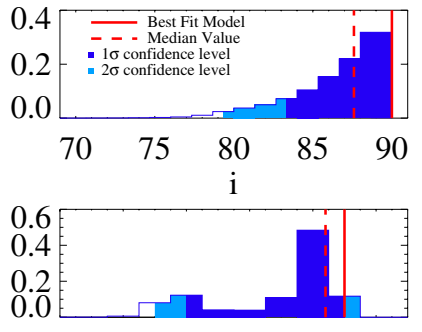

$\begin{array}{llllll}0.6 & 0.8 & 1.0 & 1.2 & 1.4 & 1.6\end{array}$

q
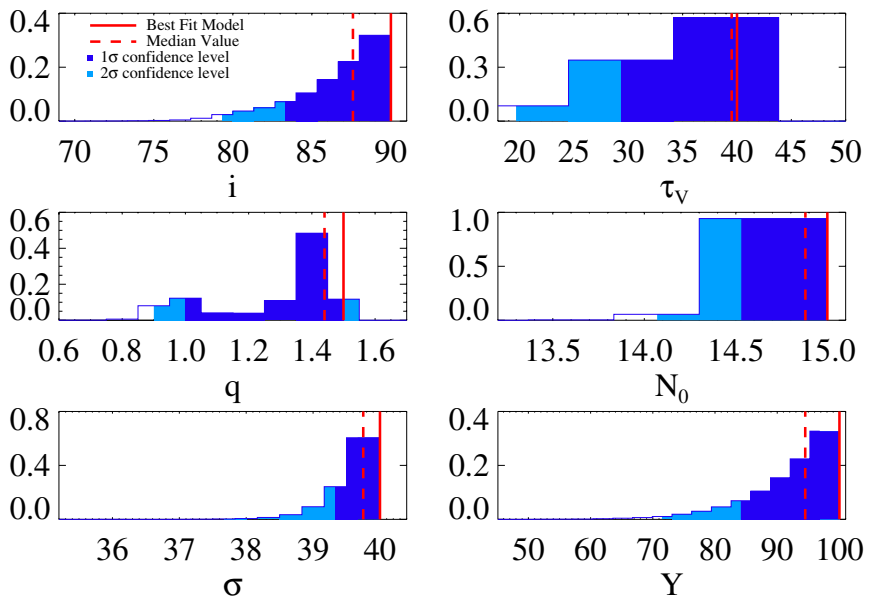

Figure 6. Histogram of the posteriors for the central spectrum plus the $18 \mu \mathrm{m}$ point with the viewing angle $i$ constrained between $50^{\circ}$ and $90^{\circ}$ and the angular thickness $\sigma$ constrained between $30^{\circ}$ and $40^{\circ}$. All plots show the value of the parameter for the best-fit model (solid line), the median value for the parameter (dashed line) and the $1 \sigma$ (dark gray) and $2 \sigma$ (light gray) confidence intervals for the parameter.

(A color version of this figure is available in the online journal.)

\subsection{Cold Dust Screen}

Due to the distance of Cyg A, even the high angular resolution of the Subaru spectra covers a larger area of the center of Cyg A (600 pc for the central spectrum). Therefore, it is possible that other components of the host galaxy contribute to the spectrum besides the torus, especially considering the depth of the silicate feature. Using the Very Long Baseline Array, Struve \& Conway (2010) found a circumnuclear H I absorption disk partially covering the nucleus and centered $\sim 80 \mathrm{pc}$ (projected) from the core. On larger scales, evidence of a dust lane was found in HST observations (Stockton et al. 1994; Jackson et al. 1998). Both of these would be contained within the Subaru central aperture. In order to test whether dust present in the host galaxy alone can account for the deep silicate feature seen in our Subaru spectra, we devised a simple model, which is composed of a blackbody (allowed to vary between 21 and $300 \mathrm{~K}$ ) covered by a cold dust screen. Since the screen in this model consists of cold dust $(<50 \mathrm{~K})$, the blackbody peak emission falls outside the wavelength range of the spectra. Therefore, the major contribution to the spectra would be absorption by the cold dust, as the emission in the MIR, including any silicate emission, would be negligible. In order to model the cold dust, we used a standard interstellar medium cross-section that was normalized to $\tau=1$ at $0.55 \mu \mathrm{m}$. The dust, chosen to match the dust composition used in CLUMPY, consists of 53\% "cold" silicates (described in Ossenkopf et al. 1992) and 47\% graphites (described in Draine 2003). Optical depth $\tau_{9.7}$ was varied in steps of 0.1 between 0 and 10 . The screened blackbody total flux was normalized to the total flux of the Subaru central spectrum. Using these temperatures and optical depths, we calculated the $\chi^{2}$ for the screened black body when compared to the Subaru central spectrum. We used the nonlinear Levenberg-Marquardt least-squares fitting method (Press et al. 2007) to calculate the best fit parameters and covariance matrix, from which the error ellipses were found (Figure 7). The best fit parameters, fitted between 7.75 and $12.5 \mu \mathrm{m}$, for the screened blackbody model for the central spectrum are $T=217 \pm 3 \mathrm{~K}$ (for the background continuum source) and $\tau_{9.7}=1.7 \pm 0.2$, with a reduced $\chi^{2}=$ 0.66 , a model probability of 0.75 , and 28 degrees of freedom. The temperature found for the fit is the temperature of a pure blackbody behind the dust screen, not the temperature of the dust itself. The $\tau_{9.7}$ fitted here is larger than that measured for the central aperture in Section 3.1. This difference is likely due to the different methods used to obtain the $\tau_{9.7}$ value, especially the simple linear spline fit used to estimate the continuum for the central aperture, which was necessary due to the limited amount of continuum emission present in the spectrum.

The cold screen model gives a much better fit than the CLUMPY model between 7.75 and $12.5 \mu \mathrm{m}$, especially in the $10 \mu \mathrm{m}$ silicate absorption feature. This suggests that foreground absorption contributes dominantly to the observed $10 \mu \mathrm{m}$ silicate absorption. A foreground screen can be included in the CLUMPY modeling as a free parameter, but the resulting models are degenerate because of the large number (seven) of free parameters. Since the cold screen model statistically gives better fits $(p$-value $=0.75$ versus $p$-value $=0.025$ for CLUMPY) of the data with only two free parameters (the blackbody temperature and the optical depth of the cold screen), it is preferred. While the CLUMPY model does fit the blue and red ends of the spectrum, and predicts the $18 \mu \mathrm{m}$ point, the overall fit is skewed by the dominance of the $10 \mu \mathrm{m}$ absorption feature. This makes it difficult to both quantify how much the torus contributes to the overall spectrum and define the properties of that torus.
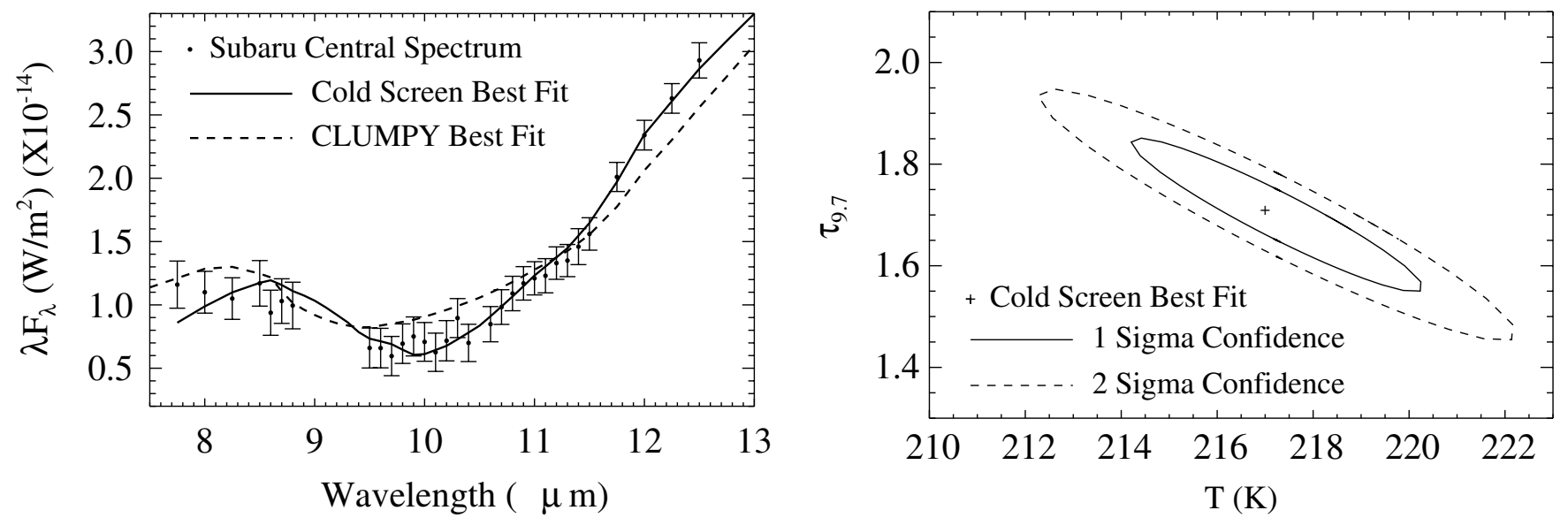

Figure 7. Left: the cold screen model (solid line) vs. CLUMPY model, fitted between 7.75 and $12.5 \mu \mathrm{m}$, (dashed line; Section 4.1), plotted over the Subaru central spectrum (dot), binned to fit the CLUMPY grid. Line features and the region affected by atmospheric ozone between 9 and $10 \mu \mathrm{m}$ have been removed from the Subaru central spectrum. Right: the $1 \sigma$ (solid line) and $2 \sigma$ (dashed line) error ellipses for the best-fit cold screen model (cross; see Section 4.2 for discussion). 
Taking the maximum temperature of the cold dust in the screen to be $50 \mathrm{~K}$, to avoid significant emission around $10 \mu \mathrm{m}$, gives a basis to determine the position of the cold screen. Temperature maps developed using high resolution MIR imaging found that temperatures of $150 \mathrm{~K}$ or higher are present up to a distance of $2 \mathrm{kpc}$ from the central engine (Radomski et al. 2002). This forces the cold screen to be further out than the central $2 \mathrm{kpc}$ of the galaxy. Therefore, the cold screen is likely made up of dust in the host galaxy that is superimposed along our line of sight to the AGN.

Since the optical depth of a foreground cold screen can be treated as the real optical depth, unlike the apparent optical depths found in Section 3.1, it is possible to estimate the hydrogen column density needed to produce such an absorption feature. Using the ratio of visual extinction to the optical depth of MIR silicate absorption (i.e., $A_{v} / \tau_{9.7}=18.5 \pm 1.5$; Roche $\&$ Aitken 1985) and the ratio of visual extinction to hydrogen column density (i.e., $A_{v} / N_{\mathrm{H}}=0.62 \times 10^{-21} \mathrm{mag} \mathrm{cm}^{-2}$; Savage \& Mathis 1979), $N_{\mathrm{H}}=(5.0 \pm 0.7) \times 10^{22} \mathrm{~cm}^{-2}$ for the cold screen model. By comparison, the X-ray hydrogen column density for the nucleus of Cyg A is $2.0 \pm 0.2) \times 10^{23} \mathrm{~cm}^{-2}$ (Young et al. 2002). The cold screen dust accounts for $\sim 25 \%$ of the column density seen in X-rays.

The above modeling favors a cold screen to explain the deep silicate feature seen in the Subaru spectra, so little can be said about the torus in Cyg A. However, in order to produce the observed absorption, there must be some source of emission behind the cold dust. The emission source should have a blackbody temperature near $217 \mathrm{~K}$, the best-fit temperature, corresponding to MIR emission by dust. Therefore, there needs to be another dust feature behind the cold screen. In addition, the emitting source must be contained within the central $600 \mathrm{pc}$ of Cyg A, since it remains unresolved in the central aperture. A torus would fulfill both of these criteria.

\subsection{Implications for Previous Modeling of Cyg A}

Previous MIR modeling of Cyg A has utilized the Spitzer spectrum as the basis for fitting a model for the torus (Privon et al. 2012). As discussed above, the $3^{\prime \prime}$ Spitzer aperture contains the inner $3 \mathrm{kpc}$ of the galaxy, for which it is impossible to disentangle contribution from the host galaxy from contribution from the AGN. In the case of Cyg A, the Spitzer spectrum and the higher spatial resolution Subaru spectra generally agree with one another at the blue and red ends of the spectra. However, the major feature in each spectra, the silicate absorption feature near $10 \mu \mathrm{m}$, shows large differences. It is this difference in the silicate absorption feature that complicates any modeling attempt. Clumpy torus models do not produce a deep silicate feature, and therefore have a difficult time reproducing the Subaru spectra. However, the larger aperture in the Spitzer spectrum allows contribution from the host galaxy to reduce the deepness of the silicate absorption, thus allowing a better fit for torus models. The better fit is misleading, as it is impossible to separate the torus from the surrounding galaxy. Cyg A provides an example of this as the Subaru spectra suggest the presence of a foreground screen of dust not associated with the torus. Therefore, it is necessary to be cautious when drawing conclusions based on torus modeling of the Spitzer spectrum only.

\section{SUMMARY}

The high angular resolution MIR spectra are dominated by the $10 \mu \mathrm{m}$ silicate absorption feature. The total and central spectra reveal deeper silicate absorption than previously seen in the Spitzer spectrum. The detection of the deep silicate feature agrees with previous MIR observations, as well as observations in other wavelength bands that detect the presence of absorbing material in the central kiloparsec of Cyg A. Since the previous spectral observation has a slit perpendicular to ours and yet has similar absorption features, the general distribution of dust around the core of Cyg A appears to peak over the nucleus. Our non-detection of any PAH features agrees with all previous spectra of Cyg A, and suggests that either the powerful central engine of Cyg A destroys the PAH, or there is not a significant star formation rate in the central kiloparsec.

Our modeling of the Subaru central spectrum suggests that absorption by foreground dust along the line of sight to the torus plays a significant, if not dominant, role in producing the deep silicate feature. The CLUMPY models cannot reproduce a deep silicate absorption feature, and the simple model of a cold dust screen agrees with previous observations of a thick dust lane in Cyg A. Therefore, it is difficult to quantify the contribution of the torus, or any of its properties, from the data. The temperature of the screened blackbody suggests the presence of MIR emission behind the screen.

Based in part on data collected at Subaru Telescope, which is operated by the National Astronomical Observatory of Japan. M.J.M. and E.S.P acknowledge support from NSF grant AST-0904890. C.P. acknowledges support from NFS grant AST0904421. M.E. acknowledges support from NFS grant AST0904316. We are pleased to acknowledge the helpful discussions with members of the Los Piratas, especially Rachel Mason and Enrique Lopez Rodriguez for intensive help with data reduction.

\section{REFERENCES}

Allen, M. G., Sparks, W. B., Koekemoer, A., et al. 2002, ApJS, 139, 411 Antonucci, R. 1993, ARA\&A, 31, 473

Asmus, D., Gandhi, P., Smette, A., Hönig, S. F., \& Duschl, W. J. 2011, A\&A, 536, A36

Burtscher, L., Jaffe, W., Raban, D., et al. 2009, ApJL, 705, L53

Burtscher, L., Meisenheimer, K., Tristram, K. R. W., et al. 2013, A\&A, 558, A149

Carilli, C. L., Bartel, N., \& Linfield, R. P. 1991, AJ, 102, 1691

Carilli, C. L., Kurk, J. D., van der Werf, P. P., Perley, R. A., \& Miley, G. K. 1999, AJ, 118, 2581

Cohen, M., Walker, R. G., Barlow, M. J., \& Deacon, J. R. 1992, AJ, 104, 1650

Cohen, M., Walker, R. G., Carter, B., et al. 1999, AJ, 117, 1864

Draine, B. T. 2003, ApJ, 598, 1017

Efstathiou, A., Hough, J. H., \& Young, S. 1995, MNRAS, 277, 1134

Efstathiou, A., \& Rowan-Robinson, M. 1995, MNRAS, 273, 649

González-Martín, O., Rodríguez-Espinosa, J. M., Díaz-Santos, T., et al. 2013, A\&A, 553, A35

Granato, G. L., \& Danese, L. 1994, MNRAS, 268, 235

Granato, G. L., Danese, L., \& Franceschini, A. 1997, ApJ, 486, 147

Hönig, S. F., Beckert, T., Ohnaka, K., \& Weigelt, G. 2006, A\&A, 452, 459

Hönig, S. F., Kishimoto, M., Gandhi, P., et al. 2010, A\&A, 515, A23

Hönig, S. F., Prieto, M. A., \& Beckert, T. 2008, A\&A, 485, 33

Imanishi, M. 2006, AJ, 131, 2406

Imanishi, M., \& Ueno, S. 2000, ApJ, 535, 626

Iye, M., Takami, H., Takato, N., et al. 2004, in Presented at the Society of PhotoOptical Instrumentation Engineers (SPIE) Conference, Vol. 5639, Society of Photo-Optical Instrumentation Engineers (SPIE) Conference Series, ed. W. Jiang \& Y. Suzuki, 1

Jackson, N., Tadhunter, C., \& Sparks, W. B. 1998, MNRAS, 301, 131 Jackson, N., Tadhunter, C., Sparks, W. B., Miley, G. K., \& Macchetto, F. 1996, A\&A, 307, L29

Jaffe, W., Meisenheimer, K., Rottgering, H. J. A., et al. 2004, Natur, 429, 47

Kataza, H., Okamoto, Y., Takubo, S., et al. 2000, Proc. SPIE, 4008, 1144

Krichbaum, T. P., Alef, W., Witzel, A., et al. 1998, A\&A, 329, 873

Krolik, J. H., \& Begelman, M. C. 1988, ApJ, 329, 702

Mason, R. E., Geballe, T. R., Packham, C., et al. 2006, ApJ, 640, 612 
Mason, R. E., Levenson, N. A., Shi, Y., et al. 2009, ApJL, 693, L136

Mason, R. E., Lopez-Rodriguez, E., Packham, C., et al. 2012, AJ, 144, 11

Mason, R. E., Ramos Almeida, C., Levenson, N. A., Nemmen, R., \& AlonsoHerrero, A. 2013, ApJ, 777, 164

Meisenheimer, K., Haas, M., Müller, S. A. H., et al. 2001, A\&A, 372, 719

Meisenheimer, K., Tristram, K. R. W., Jaffe, W., et al. 2007, A\&A, 471, 453

Nenkova, M., Ivezić, Ž., \& Elitzur, M. 2002, ApJL, 570, L9

Nenkova, M., Sirocky, M. M., Ivezić, Ž., \& Elitzur, M. 2008a, ApJ, 685, 147

Nenkova, M., Sirocky, M. M., Nikutta, R., Ivezić, Ž., \& Elitzur, M. 2008b, ApJ, 685,160

Nikutta, R., Elitzur, M., \& Lacy, M. 2009, ApJ, 707, 1550

Ogle, P. M., Cohen, M. H., Miller, J. S., et al. 1997, ApJL, 482, L37

Ossenkopf, V., Henning, T., \& Mathis, J. S. 1992, A\&A, 261, 567

Packham, C., Radomski, J. T., Roche, P. F., et al. 2005, ApJL, 618, L17

Perlman, E. S., Mason, R. E., Packham, C., et al. 2007, ApJ, 663, 808

Perlman, E. S., Sparks, W. B., Radomski, J., et al. 2001, ApJL, 561, L51

Pier, E. A., \& Krolik, J. H. 1992, ApJ, 401, 99

Pier, E. A., \& Krolik, J. H. 1993, ApJ, 418, 673

Polletta, M., Weedman, D., Hönig, S., et al. 2008, ApJ, 675, 960

Press, W. H., Teukolsky, S. A., Vetterling, W. T., \& Flannery, B. P. 2007 Numerical Recipes: The Art of Scientific Computing (3rd ed.; Cambridge: Cambridge Univ. Press)

Privon, G. C., Baum, S. A., O’Dea, C. P., et al. 2012, ApJ, 747, 46

Radomski, J. T., Packham, C., Levenson, N. A., et al. 2008, ApJ, 681, 141

Radomski, J. T., Piña, R. K., Packham, C., et al. 2003, ApJ, 587, 117

Radomski, J. T., Piña, R. K., Packham, C., Telesco, C. M., \& Tadhunter, C. N. 2002, ApJ, 566, 675
Ramos Almeida, C., Levenson, N. A., Alonso-Herrero, A., et al. 2011, ApJ, 731,92

Ramos Almeida, C., Levenson, N. A., Rodriguez Espinosa, J. M., et al 2009, ApJ, 702, 1127

Rieke, G. H., \& Low, F. J. 1972, ApJL, 176, L95

Robson, E. I., Leeuw, L. L., Stevens, J. A., \& Holland, W. S. 1998, MNRAS, 301, 935

Roche, P. F. \& Aitken, D. K. 1985, MNRAS, 215, 425

Roche, P. F., Packham, C., Telesco, C. M., et al. 2006, MNRAS, 367, 1689

Sako, S., Okamoto, Y. K., Kataza, H., et al. 2003, PASP, 115, 1407

Savage, B. D., \& Mathis, J. S. 1979, ARA\&A, 17, 73

Schartmann, M., Meisenheimer, K., Camenzind, M., et al. 2008, A\&A, 482, 67

Shi, Y., Rieke, G. H., Hines, D. C., et al. 2005, ApJ, 629, 88

Shi, Y., Rieke, G. H., Hines, D. C., et al. 2006, ApJ, 653, 127

Stockton, A., Ridgway, S. E., \& Lilly, S. J. 1994, AJ, 108, 414

Struve, C., \& Conway, J. E. 2010, A\&A, 513, A10

Tadhunter, C., Marconi, A., Axon, D., et al. 2003, MNRAS, 342, 861

Tadhunter, C. N., Packham, C., Axon, D. J., et al. 1999, ApJL, 512, L91

Tadhunter, C. N., Sparks, W., Axon, D. J., et al. 2000, MNRAS, 313, L52

Tristram, K. R. W., Meisenheimer, K., Jaffe, W., et al. 2007, A\&A, 474, 837

Tristram, K. R. W., Raban, D., Meisenheimer, K., et al. 2009, A\&A, 502, 67

Urry, C. M., \& Padovani, P. 1995, PASP, 107, 803

van der Wolk, G., Barthel, P. D., Peletier, R. F., \& Pel, J. W. 2010, A\&A, 511, A64

Young, A. J., Wilson, A. S., Terashima, Y., Arnaud, K. A., \& Smith, D. A. 2002, ApJ, 564, 176 